\title{
Interventional treatment of an aortopulmonary window due to a ruptured suture of an aortic prosthesis
}

\author{
Mareike Eißmann, MD; Philipp Kahlert, MD, FESC, FACC, FAHA, FSCAI; \\ Raimund Erbel, MD, FACC, FAHA, FESC; Rolf Alexander Jánosi*, MD, FESC \\ Department of Cardiology, West-German Heart and Vascular Center Essen, University Duisburg-Essen, Essen, Germany \\ This paper also includes supplementary data published online at: http://www.pcronline.com/eurointervention/91st_issue/192
}

An 83-year-old morbid woman presented with severe dyspnoea (New York Heart Association [NYHA] Class IV) and a history of recurrent pulmonary oedema. Due to an acute type A aortic dissection, she had previously undergone aortic surgery (January 2009), which included the implantation of an ascending aortic prosthesis.

On admission, transthoracic echocardiography revealed severe pulmonary hypertension with an estimated systolic pulmonary pressure of $70-75 \mathrm{mmHg}$ and severe tricuspid regurgitation. Using 3D transoesophageal echocardiography (Figure 1A, Moving image 1-3) and computed tomography (Figure 1B), a rupture of the aortic prosthesis with a fistula from the paraprosthetic lumen to the right pulmonary artery (Figure 1A, Figure 1B, Online Figure 1, Moving image 1-3) was found.

Because of multiple concomitant diseases and severe rightsided heart failure, an interventional approach was initiated. With a complex 3D echo-guided intervention (Online Figure 2, Moving image 4), the fistula was successfully closed using a $12 \mathrm{~mm}$
AMPLATZER $^{\text {TM }}$ ASD occluder (St. Jude Medical, St. Paul, MN, USA), which resulted in an immediate reduction in shunt volume. Post-interventional imaging showed the correct position of the ASD occluder with only minor residual flow (Online Figure 3, Moving image 5-7).

At 18-month follow-up CT (Figure 1C), the patient presented with surprising improvement of the pre-existing dyspnoea, from NYHA Class IV to NYHA Class II. The echocardiographic calculation showed a reduction in estimated systolic pulmonary pressure to $45-50 \mathrm{mmHg}$.

\section{Conflict of interest statement}

The authors have no conflicts of interest to declare.

\section{Supplementary data}

The legends of the Moving images can be found in the Online Appendix.

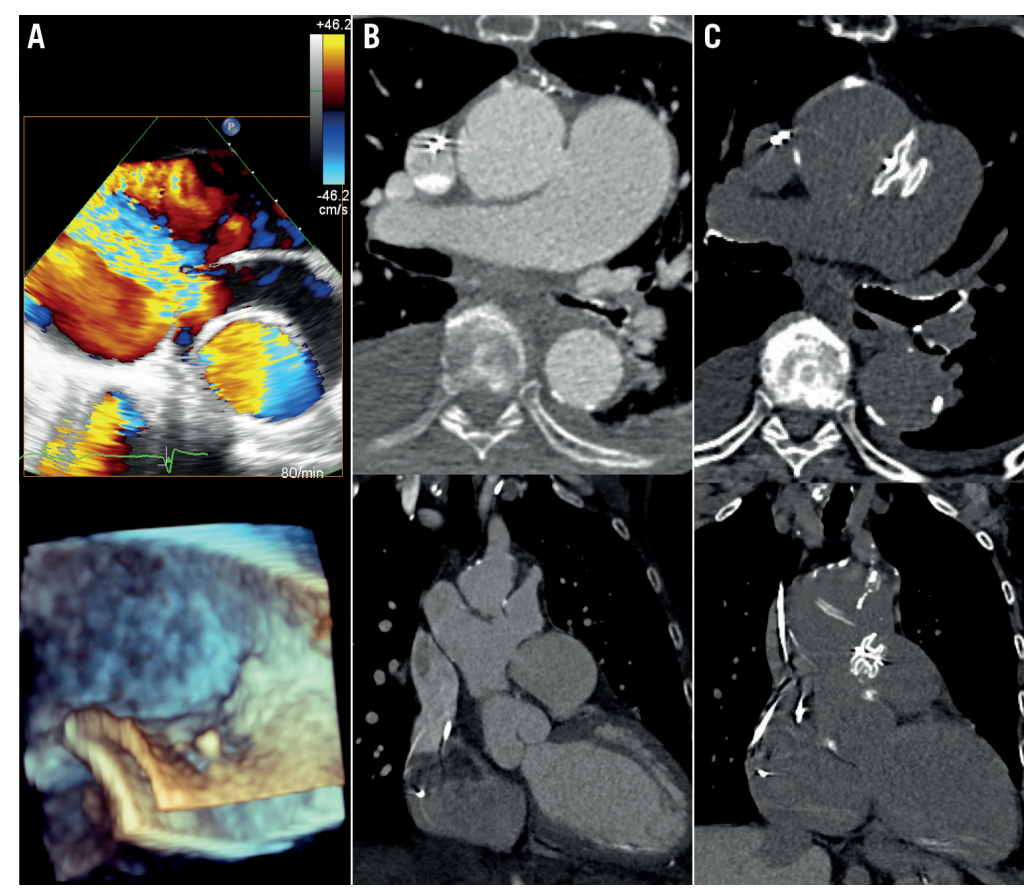

Figure 1. Aortopulmonary window. A) 3D transoesophageal echocardiography visualising a rupture of the aortic prosthesis with a fistula from the paraprosthetic lumen to the right pulmonary artery. B) Fistula visualised by computed tomography. C) Post-interventional computed tomography showing the correct position of the ASD occluder.

*Corresponding author: Department of Cardiology, West-German Heart and Vascular Center, University Hospital Essen, University of Duisburg-Essen, Hufelandstrasse 55, D-45122 Essen, Germany.E-mail: alexander.janosi@uk-essen.de 


\section{Supplementary data}

\section{Online Appendix}

Moving image 1.3D colour transoesophageal echocardiography showing a rupture of the aortic prosthesis with a fistula from the paraprosthetic lumen to the right pulmonary artery.

Moving image 2. Live 3D transoesophageal echocardiography view showing the fistula from the paraprosthetic lumen to the right pulmonary artery.

Moving image 3. 3D colour transoesophageal echocardiography showing the rupture of the aortic prosthesis with a fistula from the paraprosthetic lumen to the right pulmonary artery from a different view.

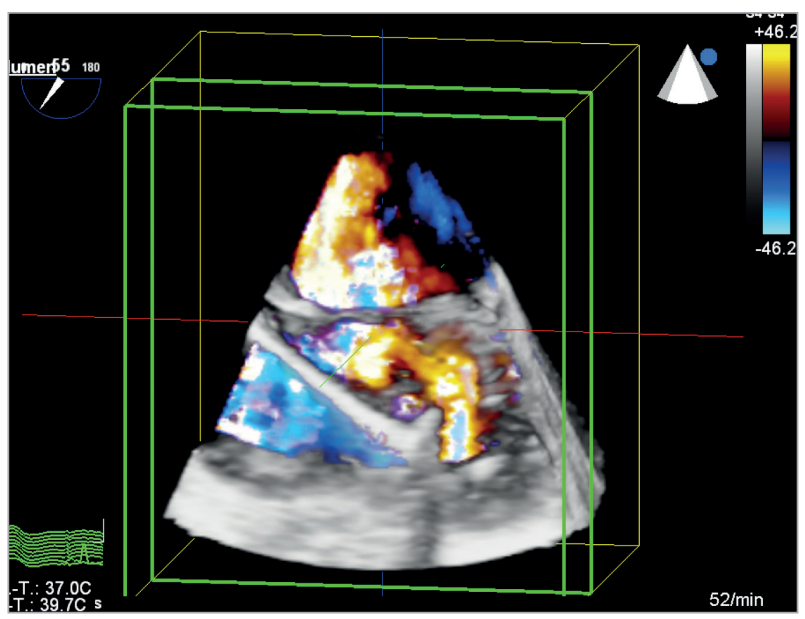

Online Figure 1. Rupture of aortic prosthesis. 3D colour transoesophageal echocardiography showing a rupture of the aortic prosthesis with a fistula from the paraprosthetic lumen to the right pulmonary artery.
Moving image 4. Angiography showing the 3D echo-guided intervention. The fistula was successfully closed using a $12 \mathrm{~mm}$ AMPLATZER ASD occluder (St. Jude Medical).

Moving image 5. Post-interventional 2D transoesophageal echocardiography showing the correct position of the ASD occluder.

Moving image 6. Post-interventional 2D colour transoesophageal echocardiography showing the correct position of the ASD occluder with only minor residual flow.

Moving image 7. Live 3D transoesophageal echocardiography en face view showing the correct position of the ASD occluder.

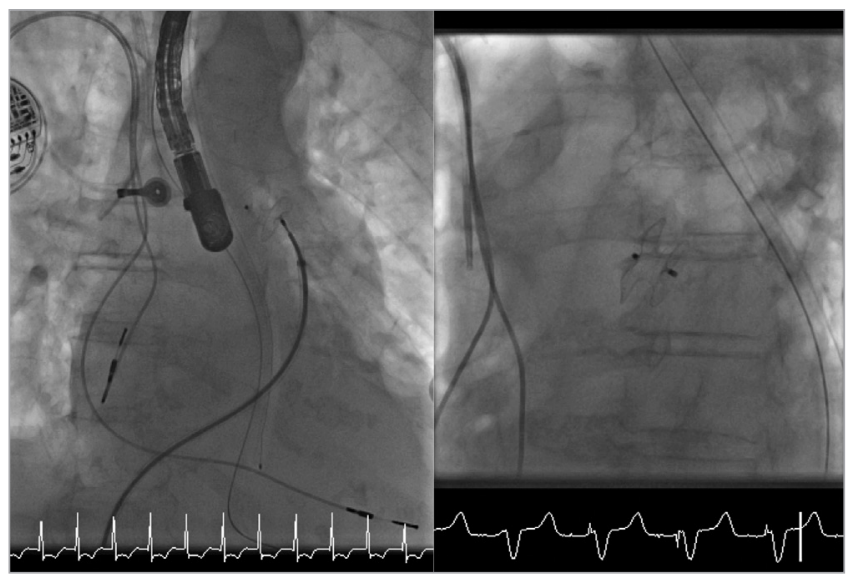

Online Figure 2. Angiographic images. 3D echo-guided intervention (left) and the correct position of the ASD occluder (right).

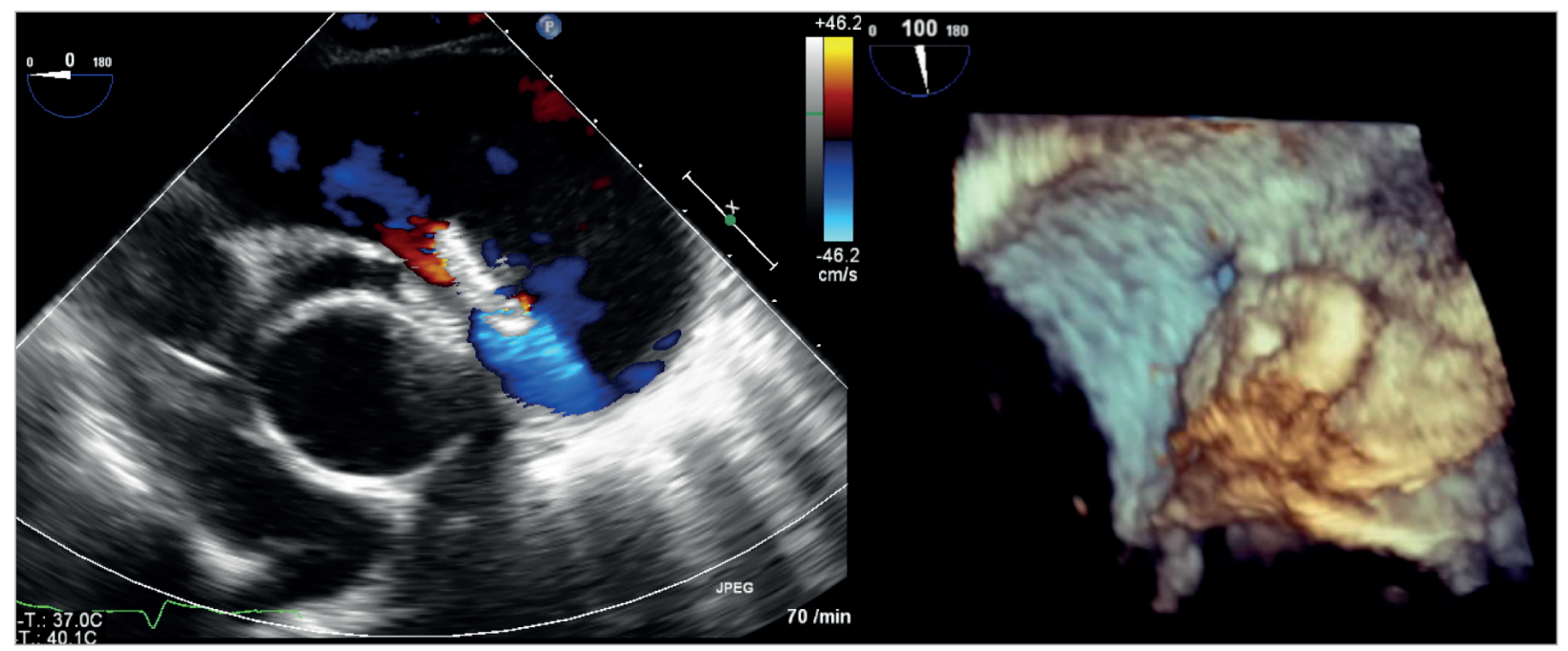

Online Figure 3. Transoesophageal echocardiography. Post-interventional 2D colour transoesophageal echocardiography showing the correct position of the ASD occluder with only minor residual flow (left). Live 3D transoesophageal echocardiography en face view showing the correct position of the ASD occluder (right). 